\title{
REAL-TIME WHITE LIGHT PSEUDOCOLOR DENSITY ENCODER
}

\author{
R. ARIZAGA, N. BOLOGNINI, H.J. RABAL, E.E. SICRE and M. GARAVAGLIA \\ Centro de Investigaciones Opticas, CONICET, 1900 La Plata, Argentine
}

Received 6 November 1981

Revised manuscript received 22 March 1982

A simple method for pseudocolor encoding of gray level information is proposed. It is based on the real-time addition of the positive and negative images of an object transparency, each one obtained in a different color. Conventional white light lamps with adequate color filters are used. Some experimental results are shown.

Pseudocoloring of gray-level information is a technique for introducing false colors to a black and white image. The importance of this operation is based on human eye better ability to distinguish different colors than gray levels.

In the last few years several analogical optical methods [1-7] have been proposed. They involve holographic techniques, half-tone screens, or spatial filtering operations for obtaining the colored image. Recently [8], we have proposed an alternative approach utilizing a modulated speckle pattern. However, the above mentioned methods are not real-time ones, and they generally involve spatial filtering steps which re-

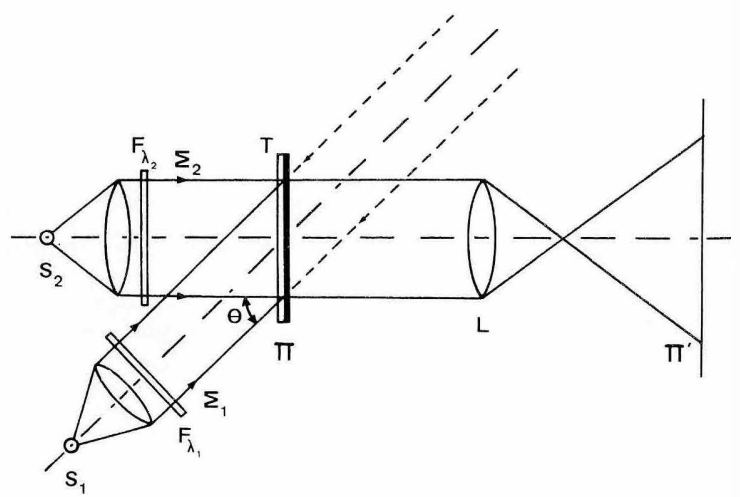

Fig. 1. Experimental set-up. $S_{1}$ and $S_{2}$ are two partially diaphragmed white light sources with color filters $F_{\lambda_{1}}$ and $F_{\lambda_{2}}$, respectively. $T$ is the object transparency placed at $\pi^{\prime}$-plane. An achromatic lens $L$ conjugates $\pi$ and $\pi^{\prime}$.

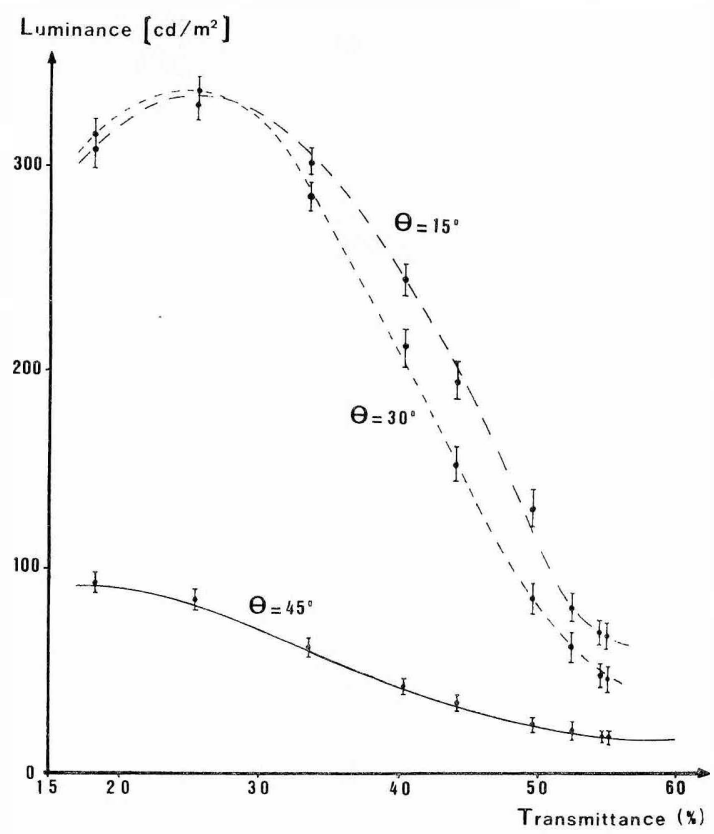

Fig. 2. The three different curves represent the luminance of the scattered light from a gray level scale, for three different $\theta$ angle values, illuminated by a $150 \mathrm{~W}$ slide projector at 30 $\mathrm{cm}$ distance. Kodak Plus-X film was used. The contrast reversal holds for lower transmittance values, as $\theta$ increases. Transmittance values were defined by neutral filters. Their error bars are negligible in the figurc scalc. 

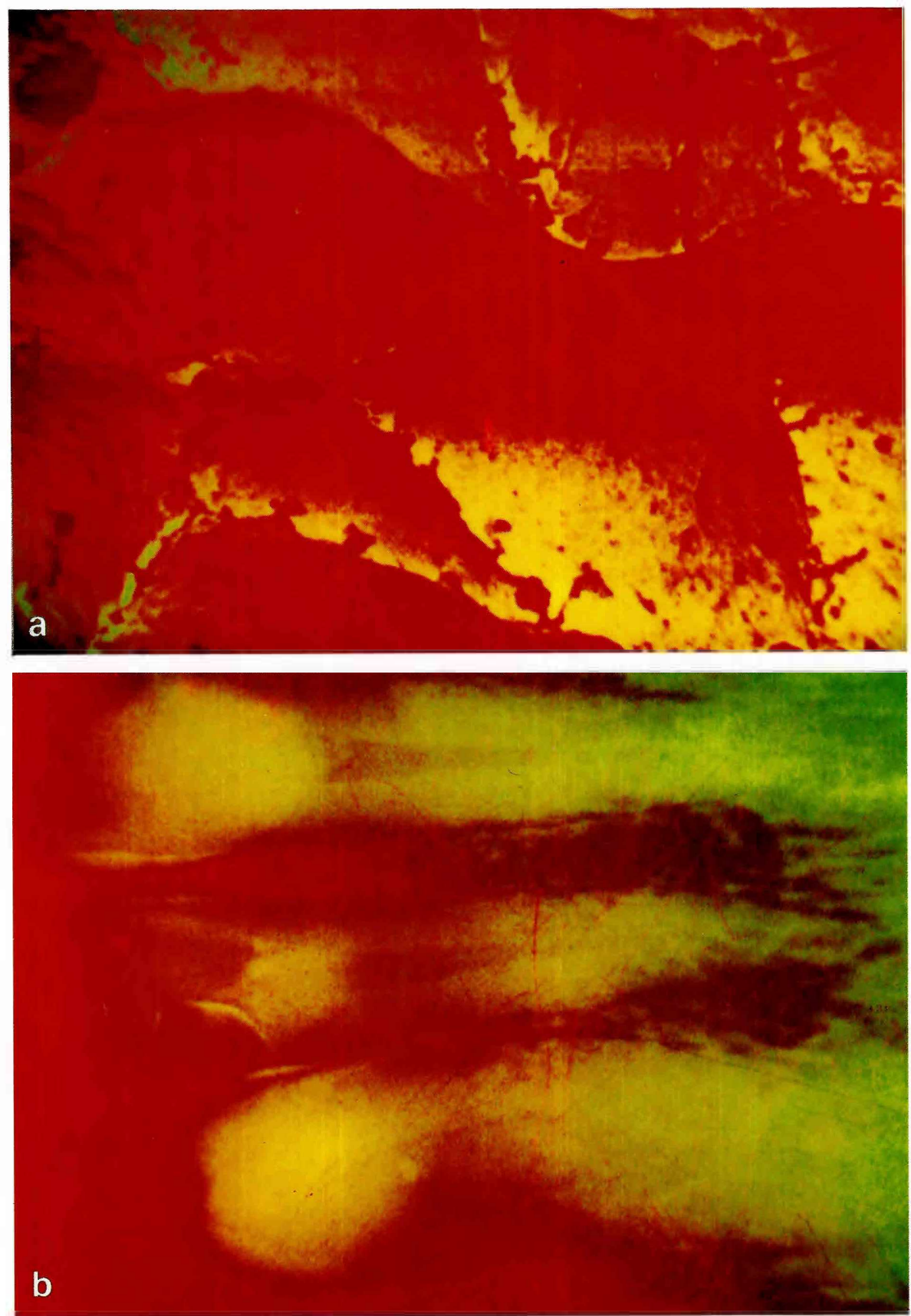

Fig. 3. (a) and (b) are pseudocolored images corresponding to transparencies (c) and (d), where (c) is a dental radiography, and (d) is a biological photomicrography. In (b) a contrast reversal saturation effect can be observed in dimmer red regions. 

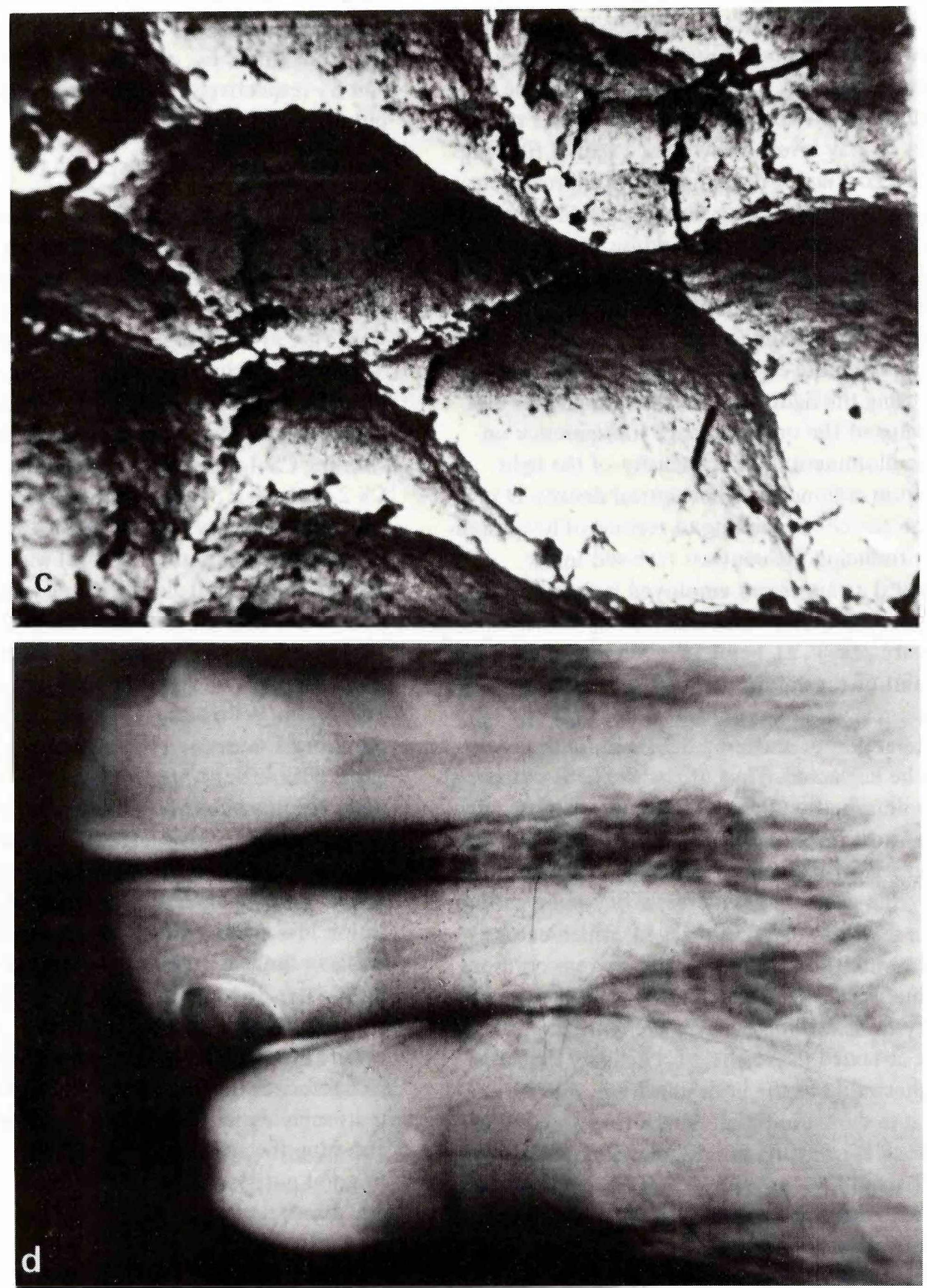
sult in a loss of spatial resolution in the final image. Santamaría et al. [9] reported a real-time pseudocoloring method, but a coherent source is still necessary. More recently, Yu et al. [10] proposed a real-time white light technique for pseudocoloring either spatial frequencies or gray levels. It involves a spatial filtering step, and the contrast reversal process is approximate.

In this paper we propose a simple method for realtime pseudocoloring of black and white transparencies. As in the case of the Santamaria's method, it is based on the simultaneous addition of a direct contrast image and a contrast reversed image, each one in a different color. In our case, the contrast reversed image is obtained by using the light scattered from the silver developed grains of the original image transparency under oblique illumination. The intensity of the light scattered from regions of higher optical density $D$ is greater than the corresponding to regions of lower density, thus producing the contrast reversed image.

The optical arrangement employed is shown in fig. 1. The object transparency $\mathrm{T}$ is described by the silver grains density $\rho(x, y, z)$, being $(x, y, z)$ the coordinates of each point of the cmulsion, and let us suppose that the maximum silver grains density is not high enough so that interaction of scattered light with other silver grains can be neglected. Thus, if a white light source $S_{1}$ is used for illuminating the transparency, as shown in fig. 1, a contrast reversed image of $\mathrm{T}$ is formed in the output plane $\pi^{\prime}$ of the achromatic lens L. However, when the value of $\rho(x, y, z)$ increases beyond a certain value $\rho_{\mathrm{L}}$, a saturation state is reached, which can be attributed to multiple scattering, and there is no more intensity increasing. Then, there exists a certain range of intensity transmittances where the contrast reversed images are obtained. Nevertheless, changing the valuc of $\theta$, a higher value of the limit density $\rho_{\mathrm{L}}$ can be attained, as it is shown in fig. 2. A way for overcoming this drawback is reversing the propagation of $\Sigma_{1}$, illuminating $\mathrm{T}$ from the opposite side of the transparency, as shown with dashed lines in fig. 1. However, due to specular reflection over the whole transparency $\mathrm{T}$, a low contrast image is obtained with this arrangement.

If another white light source $S_{2}$ illuminates $T$, in such a way that the illuminating beam $\Sigma_{2}$ propagates along the optical axis of the system, a direct contrast image is formed in the same plane $\pi^{\prime}$. Therefore, two superposed negative-positive images are simultaneously obtained, thus an intensity adjustment of both illuminating beams $\Sigma_{1}$ and $\Sigma_{2}$ can easily be done. Now, if the color filters $F_{\lambda_{1}}$ and $F_{\lambda_{2}}$ are placed in front of $S_{1}$ and $S_{2}$ respectively, the resulting intensity in the output plane $\pi^{\prime}$ becomes,

$I(x, y)=I_{\lambda_{1}}(x, y)+I_{\lambda_{2}}(x, y)$,

where $I_{\lambda_{1}}(x, y)$ is the intensity distribution of the contrast reversed image of $\mathrm{T}$ in the color given by $\lambda_{1}$, and $I_{\lambda_{2}}(x, y)$ is the corresponding intensity of the direct contrast image of $\mathrm{T}$ in the color given by $\lambda_{2}$. In this way, $I(x, y)$ describes a pseudocolored image of $\mathrm{T}$.

Some experimental results are shown in fig. 3. Two identical projectors $(150 \mathrm{~W})$ were used as illuminating sources $\mathrm{S}_{1}$ and $\mathrm{S}_{2}$. The color filters employed were Corning CS 4-65 (green, $x_{\mathrm{A}}=0.40, y_{\mathrm{A}}=0.55$ ) and CS 2-60 (red, $\left.x_{\mathrm{A}}=0.72, y_{\mathrm{A}}=0.28\right)$. To obtain an adequate intensity balance at the output plane $\pi^{\prime}$, a neutral density filter (N.D. $=2$ ) was used to attenuate $\Sigma_{2}$. Also, a ground glass diffuser was placed in the $\Sigma_{2}$ path to provide uniform illumination to the transmitted image. Duc to the color filters cmployed, intermediate gray levels were assigned to the different hues in the yellow-orange region. In fig. $3 \mathrm{~b}$ the above mentioned saturation effect is clearly observed in regions with lower transmittance, where almost the same color is obtained for different gray levels.

We have presented a real-time white light processing for pseudocolor encoding of gray levels. Because no spatial filtering operation is required, there is no resolution loss in the colored image, regardless of the diffraction limit imposed by the optical system. As in most cases of white light pseudocolor techniques, two color filters are needed. In this way, as the coding colors are not spectral ones, the additive mixture that characterizes the several gray-levels has a purity that is strongly dependent on the filters employed. By choosing the appropriate color filters, a broad range of good purity colors can be obtained, so improving eye discrimination.

The authors wish to thank the Secretaría de Estado de Ciencia y Tecnología, Argentina, and the Organization of American States, for providing financial support for this work. 


\section{References}

[1] H.K. Liu and J.W. Goodman, Nouv. Rev. d'Opt. 7 (1976) 285.

[2] G. Indebetouw, Appl. Optics 16 (1977) 1951.

[3] A. Tai, F.T.S. Yu and H. Chen, Optics Lett. 3 (1978) 190.

[4] F.T.S. Yu, Optics Lett. 3 (1978) 57.

[5] F.T.S. Yu, A. Tai and H. Chen, J. Optics 9 (1978) 269.

[6] T.H. Chao, S.L. Zhuang and F.T.S. Yu, Optics Lett. 5 (1980) 230.
[7] S. Guel Sandoval, J. Santamaría and J.H. Altamirano, Optics in Four Dimensions, Ensenada, México, August (1980).

[8] H.J. Rabal, E.E. Sicre, N. Bolognini and M. Garavaglia, Optics Comm., 39 (1981) 225.

[9] J. Santamaría, M. Gea and J. Bescós, J. Optics 10 (1979) 151.

[10] F.T.S. Yu, S.L. Zhuang, T.H. Chao and M.S. Dymek, Appl. Optics 19 (1980) 2986. 